\title{
Effective magnetic field for photons based on the magneto-optical effect
}

\author{
Kejie Fang* \\ Department of Physics, Stanford University, Stanford, California 94305, USA \\ Shanhui Fan \\ Department of Electrical Engineering, Stanford University, Stanford, California 94305, USA
}

(Received 4 July 2013; published 31 October 2013)

\begin{abstract}
We propose to create an effective magnetic field for photons in photonic crystal resonator lattices using the magneto-optical effect. The inter-resonator coupling is mediated by magneto-optical waveguides or magnetooptical resonators, and thus the coupling between the nearest-neighbor photonic crystal resonators acquire a direction-dependent phase. The effective magnetic field can be realized with a proper choice of the spatial distribution of such a direction-dependent phase.
\end{abstract}

DOI: 10.1103/PhysRevA.88.043847

PACS number(s): 42.70.Qs, 85.70.Sq

\section{INTRODUCTION}

Gauge fields in Nature are associated with charged particles. Photons are neutral particles and thus there are no naturally existing gauge fields that couple to photons. Nevertheless, the concepts of effective gauge fields and effective magnetic field for photons have attracted wide interest in recent years [1-8]. The effective gauge fields for photons can generate novel photonic phenomena, such as photonic analogs of the quantum Hall effect [4], the quantum spin Hall effect [6,8], and the de Haas-van Alphen effect [9]. The effective magnetic field that breaks time-reversal symmetry can also induce nonreciprocal flow of light, and provides great flexibility in tailoring light propagation in general $[9,10]$. Moreover, the development of effective gauge fields for photons are generally connected with studies into topological properties associated with photonic systems [11-13].

Several mechanisms have been proposed for generating effective magnetic fields for photons. References [1,2,6-8] create effective magnetic fields that are related to a certain type of spin degrees of freedom of light or strains in photonic lattices [7], and as a result time-reversal symmetry is conserved in these systems. In contrast, the scheme of Refs. [3,4] based on dynamic modulation [14,15] explicitly breaks time-reversal symmetry, and thus the effective magnetic field generated there also breaks time-reversal symmetry. Consequently, this effective magnetic field can be applied to achieve nonreciprocal light propagation effects such as the robust one-way edge mode [4].

Instead of using the dynamic modulation of dielectric constant, the conventional way to break time-reversal symmetry for photons is through the use of the magneto-optical effect [16]. The magneto-optical effect has been used to achieve effective magnetic field for photons in momentum space through a connection with Berry's curvature $[11,12,17,18]$. In contrast, in this paper, we propose using the magneto-optical effect to create effective magnetic field for photons in real space.

The paper is organized as follows. In Sec. II, we review the general requirement to achieve an effective magnetic field for

\footnotetext{
*Present address: Thomas J. Watson, Sr., Laboratory of Applied Physics, California Institute of Technology, Pasadena, CA 91125, USA.
}

photons in real space. In Sec. III, we propose an implementation to meet this requirement in a photonic crystal resonator lattice, where the coupling of resonators is mediated through magneto-optical waveguides. In Sec. IV, we provide an alternative implementation in a photonic crystal resonator lattice where the coupling between resonators is mediated by magneto-optical resonators. The use of such mediating magneto-optical resonators results in miniaturization of the structure and enhancement of the nonreciprocity. We conclude in Sec. V.

\section{GENERAL PRINCIPLE FOR CREATING EFFECTIVE MAGNETIC FIELD}

We first review the general principle for creating an effective magnetic field for photons in a resonator lattice $[3,4]$. The idea is to seek to create a photonic Hamiltonian that emulates the electronic tight-binding model under a real magnetic field.

In the absence of magnetic fields, the Hamiltonian of a tightbinding model describing noninteractive electrons generally has the following form:

$$
H=\sum_{r^{\prime}, r} V_{r^{\prime}, r}^{0} b_{r^{\prime}}^{\dagger} b_{r}+\text { H.c. },
$$

where $b_{r}^{\dagger}\left(b_{r}\right)$ is the creation (annihilation) operator at site $r$, $V_{r^{\prime}, r}^{0}$ is the coupling coefficient that describes hopping between sites $r$ and $r^{\prime}$, and we have suppressed the label for the spin of electrons. In the presence of a magnetic field, associated with a gauge field $\vec{A}$, the Hamiltonian is modified via Peierls' substitution [19]:

$$
H^{\prime}=\sum_{r^{\prime}, r} e^{i \phi_{r^{\prime}, r}} V_{r^{\prime}, r}^{0} b_{r^{\prime}}^{\dagger} b_{r}+\text { H.c. }
$$

with

$$
\phi_{r^{\prime}, r}=\frac{q_{e}}{\hbar} \int_{r}^{r^{\prime}} \vec{A} \cdot d \vec{l}
$$

where $q_{e}$ is the electron charge and $\hbar$ is the reduced Planck constant. Note that this phase is direction dependent, since $\phi_{r, r^{\prime}}=\frac{e}{\hbar} \int_{r^{\prime}}^{r} \vec{A} \cdot d \vec{l}=-\phi_{r^{\prime}, r}$. Thus, for a general tight-binding model of the form of Eq. (2), the phase $\phi_{r^{\prime}, r}$ in the hopping coefficient $V_{r^{\prime}, r} \equiv e^{i \phi_{r^{\prime}, r}} V_{r^{\prime}, r}^{0}$ is associated with the gauge field. 


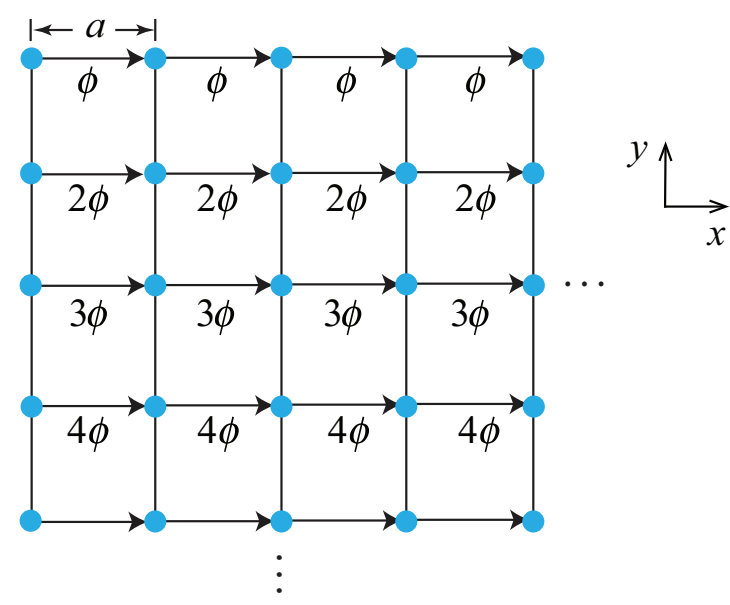

FIG. 1. (Color online) A scheme of photonic resonator lattice with uniform effective magnetic field. The direction-dependent coupling phases (along positive $x$ ) on the $x$ bonds are labeled and on the $y$ bonds are zero.

The magnetic field is related to the phase of the hopping coefficient through

$$
B=\frac{\hbar}{q_{e} a^{2}} \sum \phi_{r^{\prime}, r},
$$

where the summation is over all the bonds on the boundary of unit cell following the counterclockwise hopping direction and $a^{2}$ is the area of unit cell. For simplicity, we have assumed a square lattice with $a$ the distance between two nearest-neighbor resonators.

A convenient phase distribution corresponding to a spatially uniform magnetic field of strength $-\hbar \phi_{0} /\left(q_{e} a^{2}\right)$ is the socalled Landau gauge:

$$
\begin{gathered}
\phi_{r, r+a \hat{x}}=\frac{\phi_{0}}{a} y, \\
\phi_{r, r+a \hat{y}}=0,
\end{gathered}
$$

where $\hat{x}(\hat{y})$ is the unit vector along the $x(y)$ axis (Fig. 1).

The idea for creating an effective magnetic field for photons is to implement a photonic resonator lattice that is described by the Hamiltonian of Eq. (2). In such a photonic resonator lattice, we can then define an effective gauge field and effective magnetic field relating to the direction-dependent hopping phase through the following equations $[3,4]$ :

$$
\begin{gathered}
\vec{A}_{\text {eff }}=\frac{\phi_{r^{\prime}, r}}{a} \hat{l}, \\
B_{\text {eff }}=\frac{\sum \phi_{r^{\prime}, r}}{a^{2}},
\end{gathered}
$$

where $\phi_{r^{\prime}, r}$ is the direction-dependent phase acquired by photons from $r$ to $r^{\prime}$ and $\hat{l}$ is the unit vector pointing from $r$ to $r^{\prime}$, and the summation in Eq. (8) is over all the bonds on the boundary of the unit cell following the counterclockwise hopping direction.

In Refs. [3,4], the direction-dependent phase for photons is achieved through the photonic transition induced by dynamic modulations. And by properly assigning the spatial distribution of the modulation phases, we can achieve the Hamiltonian of
Eq. (2) in the Landau gauge. In this paper we instead use the direction-dependent phase created by the magneto-optical effect in order to realize an effective magnetic field for photons. Unlike the dynamic modulation schemes, magneto-optics is purely passive and does not require energy input, but does present a very different set of challenges in material processing and on-chip integration as compared with modulation. Therefore, it would be of interest to pursue the generation of effective magnetic field for photons using the magneto-optical effect.

In the rest of the paper, we provide two different schemes to realize the direction-dependent phase based on the magnetooptical effect, and use them to achieve the Hamiltonian of Eq. (2) in the Landau gauge and thus to create effective magnetic field for photons. Our main focus is to show that an effective magnetic field for photons can arise from various magneto-optical effects in waveguides and resonators. The properties of such magneto-optical waveguides and resonators are well known in the literature. Therefore, for the purpose of conciseness, we do not in this paper present a detailed numerical design of waveguides and resonators, but rather rely upon the literature to obtain estimations that are relevant for discussions of the properties of effective magnetic fields.

\section{WAVEGUIDE-MEDIATED COUPLING SCHEME}

In this section, we implement the photonic resonator lattice with uniform effective magnetic field as schematically shown in Fig. 1 using photonic crystal resonators and magneto-optical waveguides. The direction-dependent coupling between photonic crystal resonators are achieved by a mediating magnetooptical waveguide. We will start from the discussion of modal properties of a single magneto-optical waveguide, and then derive the coupling between two resonators mediated by a magneto-optical waveguide, and finally obtain an effective magnetic field in a two-dimensional lattice.

\section{A. Magneto-optical waveguide}

We consider a magneto-optical waveguide in a photonic crystal made of square lattice of air holes in a silicon slab [Fig. 2(a)]. The magneto-optical waveguide is achieved by creating a line defect consisting of an array of missing air holes, and by bonding a bar of magneto-optic material on top of this line defect. In general, assuming that magnetization is along the $y$ direction, the dielectric response of the magneto-optical material is then described by a tensor

$$
\epsilon=\left(\begin{array}{ccc}
\epsilon_{r} & 0 & i \epsilon_{i} \\
0 & \epsilon_{r} & 0 \\
-i \epsilon_{i} & 0 & \epsilon_{r}
\end{array}\right),
$$

where $\epsilon_{r}$ is the dielectric constant of the material without the magneto-optical effect and $\epsilon_{i}$ is the off-diagonal component due to the magneto-optical effect, which is proportional to the magnitude of magnetization field $M$. We consider the transverse magnetic ( $H$ field in $x-y$ plane) mode of the waveguide. In the absence of magnetization in the magneto-optical bar, transverse magnetic modes propagating along positive and negative $x$ directions are degenerate, i.e., they have the 
(a)
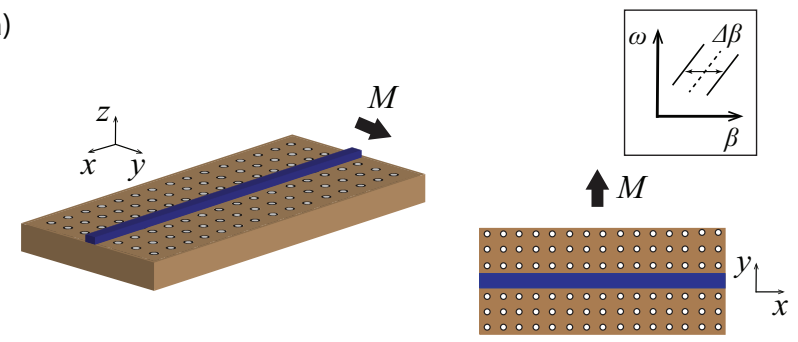

(b)
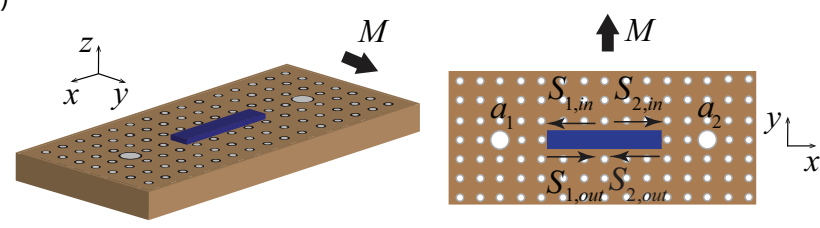

(c)

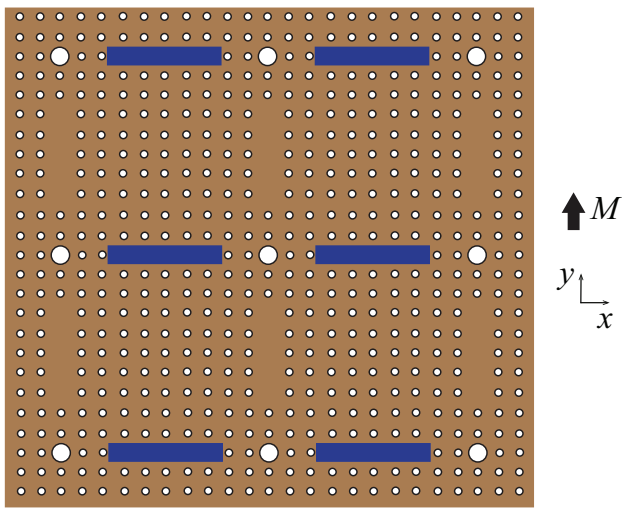

FIG. 2. (Color online) (a) Three-dimensional view (left) and top view (right) of a magneto-optical photonic crystal waveguide. The magnetization $M$ is along $y$ axis. Inset is a schematic plot of dispersion splitting of the forward and backward propagating waveguide modes. (b) Three-dimensional view (left) and top view (right) of two photonic crystal resonators coupled through an intermediate magneto-optical waveguide. (c) Top view of a square lattice of photonic crystal resonators with magneto-optical waveguide-mediated coupling along $x$ axis and nonmagneto-optical waveguide-mediated coupling along $x$ axis.

same wave vector for a same frequency, due to time-reversal symmetry. In the presence of a nonzero magnetization in the magneto-optical material along the $y$ axis, the degeneracy between the two counterpropagating modes splits since the time-reversal symmetry is broken. Assuming that $H_{y}$ is the dominant magnetic-field component of these modes, the splitting of the wave vectors of two modes at a given frequency is given by [20]

$$
\Delta \beta=\frac{\int H_{y}(x)^{2} \partial_{x}\left(\epsilon_{i} / \epsilon_{r}^{2}\right) d x}{\int \epsilon_{r}^{-1}\left|H_{y}(x)\right|^{2} d x} .
$$

This wave-vector splitting results in a difference in phase shifts as waves propagate along forward or backward directions, i.e., $\phi_{L}-\phi_{R}=\Delta \beta L$, where $L$ is the length of the waveguide. The phase difference is thus proportional to the magnetization $M$ through Eq. (10) as $\epsilon_{i}$ is proportional to $M$.

Experimentally, using a rib-waveguide geometry where the modes are weakly confined, a nonreciprocal phase difference of $21 \mathrm{rad} / \mathrm{cm}$ has been measured experimentally in Ref. [21], using Ce-substituted yttrium iron garnet as the magneto-optical materials. By strong confinement of the waveguide modes through slot waveguide geometry, a phase shift as high as $1000 \mathrm{rad} / \mathrm{cm}$ has been reported in numerical simulations [22]. Also, as seen from Eq. (10), $\Delta \beta$ is proportional to $\epsilon_{x z}$, and thus is proportional to the magnetization strength. As a result, the phase-shift difference is also proportional to magnetization strength, which in practice can be controlled by varying the strength of applied external magnetic field as long as the magnetization is not saturated.

\section{B. Direction-dependent coupling between two resonators mediated by a magneto-optical waveguide}

Using the properties of transverse magnetic waveguide modes under the magneto-optical effect as discussed above, we now derive the direction-dependent coupling between two photonic crystal resonators mediated by a magneto-optical waveguide. The structure is shown in Fig. 2(b), where a photonic resonator is created by enlarging an air hole. Each resonator supports a singly degenerate resonant mode of frequency $\omega_{0}$ (we note that any singly degenerate resonator is sufficient for our purpose here). They couple to the transverse magnetic modes of the intermediate magneto-optical waveguide by evanescent fields. The magnetization is along $y$ axis.

Suppose the field amplitudes of the two resonators are $a_{1(2)}$, and the incoming and outgoing fields of the two resonators through the waveguide are $S_{1(2) \text {,in }}$ and $S_{1(2) \text {,out }}$ respectively. Then the coupled-mode equations for the two resonators are

$$
\begin{gathered}
\frac{d a_{1}}{d t}=-\left(i \omega_{0}+\frac{\gamma}{2}\right) a_{1}+\sqrt{\gamma} S_{1, \text { in }}, \\
S_{1, \text { out }}=-S_{1, \text { in }}+\sqrt{\gamma} a_{1}, \\
\frac{d a_{2}}{d t}=-\left(i \omega_{0}+\frac{\gamma}{2}\right) a_{2}+\sqrt{\gamma} S_{2, \text { in }}, \\
S_{2, \text { out }}=-S_{2, \text { in }}+\sqrt{\gamma} a_{2},
\end{gathered}
$$

where $\gamma$ is the energy decay rate of the resonators to the waveguide mode, which is the only decay channel we assumed here. From now on, we will work in the rotating frame by making the substiution $a_{1(2)} \rightarrow a_{1(2)} e^{-i \omega_{0} t}$, which eliminates the terms $-i \omega_{0} a_{1(2)}$ from the coupled-mode equations.

The incoming and outgoing fields in Eqs. (11)-(14) are the propagating modes of the waveguide, therefore, we have the relations

$$
\begin{aligned}
& S_{2, \text { in }}=e^{i \phi_{R}} S_{1, \text { out }}, \\
& S_{1, \text { in }}=e^{i \phi_{L}} S_{2, \text { out }} .
\end{aligned}
$$

As discussed in Sec. III A, $\phi_{R}$ and $\phi_{L}$ can be made different using the magneto-optical effect. Substituting Eqs. (15) and (16) into Eqs. (11)-(14) and eliminating the waveguide modes, 
we obtain an effective coupling between the two resonators governed by the following equations:

$$
\begin{aligned}
& \frac{d a_{1}}{d t}=-\frac{\gamma}{2} \frac{1+e^{i\left(\phi_{R}+\phi_{L}\right)}}{1-e^{i\left(\phi_{R}+\phi_{L}\right)}} a_{1}+i V_{12} a_{2}, \\
& \frac{d a_{2}}{d t}=-\frac{\gamma}{2} \frac{1+e^{i\left(\phi_{R}+\phi_{L}\right)}}{1-e^{i\left(\phi_{R}+\phi_{L}\right)}} a_{2}+i V_{21} a_{1},
\end{aligned}
$$

where

$$
\begin{aligned}
& V_{12}=e^{i\left(\phi_{L}-\phi_{R}\right) / 2} \frac{\gamma}{2 \sin \frac{\phi_{L}+\phi_{R}}{2}}, \\
& V_{21}=e^{i\left(\phi_{R}-\phi_{L}\right) / 2} \frac{\gamma^{2}}{2 \sin \frac{\phi_{L}+\phi_{R}}{2}} .
\end{aligned}
$$

With proper choice of the waveguide length, one can realize $\phi_{L}+\phi_{R}=\pi(\bmod 2 \pi)$. In such a case, the first terms on the right-hand side of Eqs. (17) and (18) are pure imaginary and represent a frequency renormalization of the two resonators due to coupling to the waveguide modes. From Eqs. (19) and (20), we also see that the effective coupling coefficient between two resonators acquires a direction-dependent phase shift of the magnitude of $\left(\phi_{L}-\phi_{R}\right) / 2$.

\section{Two-dimensional resonator lattice}

Having shown that we can achieve a direction-dependent phase shift in the coupling between two resonators, we now construct a resonator lattice shown in Fig. 2(c) to create an effective magnetic field for photons. In this structure, nearestneighbor photonic crystal resonators along the $x$ axis are connected by magneto-optical waveguides magnetized along the $y$ axis, while nearest-neighbor photonic crystal resonators along the $y$ axis are connected by waveguides without bonding magneto-optical material.

According to Eqs. (19) and (20), the coupling coefficient from resonators at $r=(x, y)$ to the resonator at $r+a \hat{y}$ is given by

$$
V_{r, r+a \hat{y}}=\frac{\gamma}{2},
$$

and the coupling coefficient from the resonator at $r$ to the resonator at $r+a \hat{x}$ is given by

$$
V_{r, r+a \hat{x}}=e^{i \phi(y)} \frac{\gamma}{2},
$$

where $\phi(y)=\phi_{L}(y)-\phi_{R}(y)$. In order to implement the Landau gauge of Eq. (5), the magnetization is designed to have a gradient along the $y$ axis, i.e., $M(y)=M_{0} y / a$, which results in $\phi(y)=\phi_{0} y /\left(N_{y} a\right)$, where $\phi_{0}$ is a constant and $N_{y}+1$ is the number of resonators along the $y$ axis [23]. Equations (21) and (22) together implement the Landau gauge of a uniform effective magnetic field with strength

$$
B_{\text {eff }}=\frac{\phi_{0}}{N_{y} a^{2}} .
$$

If one makes a lattice of Fig. 2(c) with waveguide length of $a=10 \mu \mathrm{m}$ and $N_{y}=100$, then $B_{\text {eff }} \approx 0.001 / a^{2}$, where we used a maximum nonreciprocal phase shift of $100 \mathrm{rad} / \mathrm{cm}$, which is about an order of magnitude smaller than that of Ref. [22]. To obtain a sense of the magnitude of the effective magnetic field, we note that the radius of the trajectory of a Gaussian beam with momentum $k(|k| \ll \pi / a)$ under the effective magnetic field is given by $R=k / B_{\text {eff }}$ [4]. For $k=0.1 / a$ and $B_{\text {eff }} \approx 0.001 / a^{2}$, we have $R \approx 100 a$, which is comparable to the total size of the lattice. Therefore, one should be able to use such a lattice to observe Lorentz force for photons, in a setup similar to Ref. [4].

\section{RESONATOR-MEDIATED COUPLING SCHEME}

In this section, we consider another realization of the photonic resonator lattice with a uniform effective magnetic field, where the direction-dependent coupling between two photonic crystal resonators are realized by an intermediate magneto-optical resonator. Such a configuration enables miniaturization of the intermediate coupling part through resonance enhancement of nonreciprocity. In this case, the degeneracy between even and odd resonant modes of the intermediate resonator splits under a normal magnetization due to the magneto-optical effect. The resonant frequency splitting, as we will show, leads to an enhanced direction-dependent phase for photons.

\section{A. Magneto-optical resonator}

Consider a photonic crystal of square lattice of air holes in a silicon chip, one can make a photonic crystal resonator by eliminating one air hole [Fig. 3(a)]. We consider the transverse electric modes ( $E$ field only has in-plane components) of the photonic crystal. The resonator supports two degenerate even $(|e\rangle)$ and odd $(|o\rangle)$ dipolar modes (with respect to the $y$ axis) with frequency $\omega_{0}$ [24]. If we bond a magneto-optical material on top of the resonator and apply a magnetization perpendicular to the planar structure, the magneto-optical effect splits the degeneracy of the even and odd modes and mixes them into clockwise $(|e\rangle-i|o\rangle)$ and counterclockwise $(|e\rangle+i|o\rangle)$ rotating modes, with frequency $\omega_{L}$ and $\omega_{R}$ respectively. For a weak magneto-optical effect, the frequency splitting $\Delta \omega=\left|\omega_{R}-\omega_{L}\right|$ can be calculated perturbatively and is given by [25]

$$
\Delta \omega=\frac{\omega_{0} \int d S \epsilon_{i} \vec{z} \cdot\left(\vec{E}_{e}^{*} \times \vec{E}_{o}\right)}{\sqrt{\int d S \epsilon_{r}\left|\vec{E}_{e}\right|^{2} \int d S \epsilon_{r}\left|\vec{E}_{o}\right|^{2}}},
$$

where $\vec{E}_{e(o)}$ is the electric field of the even (odd) mode. In this case with the magnetization along the $z$ direction, the dielectric tensor has the same form as Eq. (9) except that the term $i \epsilon_{i}$ now appears as $\epsilon_{x y}$. We find $\Delta \omega \propto \epsilon_{i}$, and thus is proportional to the magnetization $M$. To achieve large $\Delta \omega\left(\sim \omega_{0} \epsilon_{i} / \epsilon_{r}\right)$, a magnetic domain engineering is generally needed [25]. To avoid the magnetic domain engineering while still achieving large frequency splitting, one can nevertheless design a more sophisticated resonator and thus using uniform magnetization [26].

\section{B. Direction-dependent coupling between two resonators mediated by a magneto-optical resonator}

We derive the direction-dependent coupling between two resonators in the photonic crystal mediated by a magnetooptical resonator. The structure is shown in Fig. 3(b), where photonic crystal resonators are created by expanding the air holes and connected by a short section of waveguide 
(a)

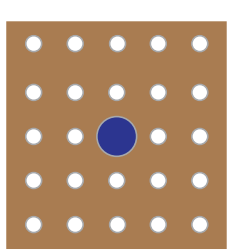

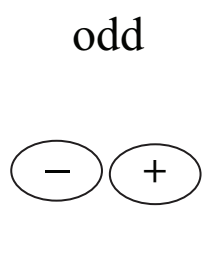

(b)

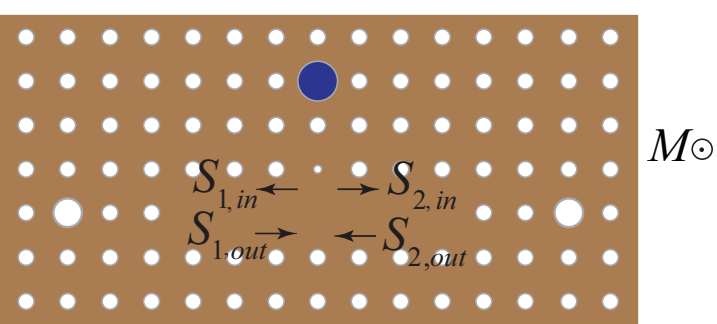

(c)

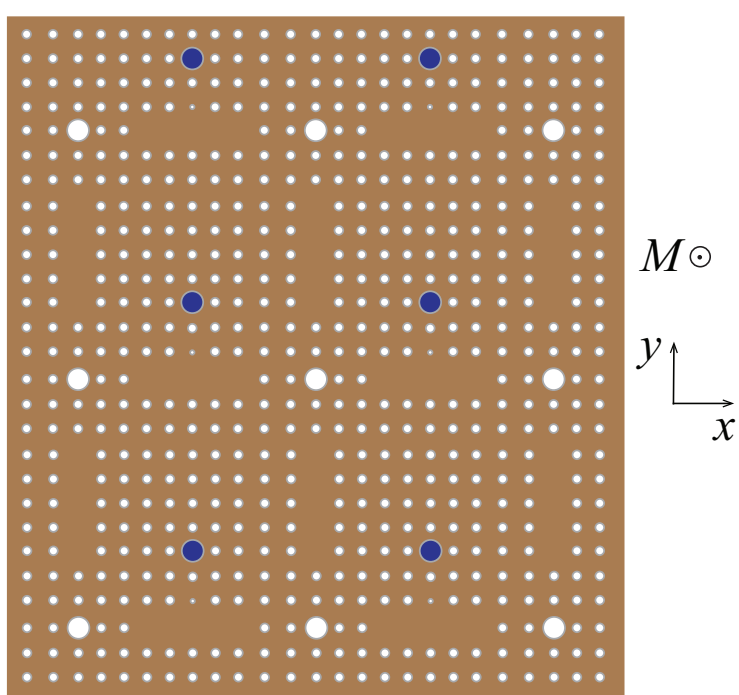

FIG. 3. (Color online) (a) A photonic crystal resonator which supports two degenerate even and odd dipolar modes (schematic) bonded with magneto-optical material (blue). (b) Light transmission through a photonic crystal waveguide side-coupled to a magnetooptical resonator (blue). The magnetization $M$ is along $z$ axis. (c) A square lattice of photonic crystal resonators (red) with magnetooptical resonator-mediated coupling along $x$ axis.

side-coupled to a magneto-optic resonator [27]. The coupledmode equations are the same as Eqs. (11)-(16), except that the direction-dependent phases $\phi_{R}$ and $\phi_{L}$ are now determined by the resonant frequency splitting of the magneto-optical resonator.

To see the direction-dependent phase, we consider the light transmission through the waveguide side-coupled to the magneto-optical resonator. For the light propagating to the right, it couples to the counterclockwise rotating resonance of the resonator as required by symmetry matching between the incoming wave and the localized resonance [24,28]. By solving the coupled-mode equation for the magneto-optic resonator,

$$
\begin{gathered}
\frac{d a_{c c}}{d t}=-\left(i \omega_{R}+\frac{\gamma_{r}}{2}\right) a_{c c}+i \sqrt{\gamma_{r}} S_{1, \mathrm{out}}, \\
S_{2, \text { in }}=S_{1, \mathrm{out}}+i \sqrt{\gamma_{r}} a_{c c},
\end{gathered}
$$

the transmission relation is given by

$$
S_{2, \text { in }}=\frac{i\left(\omega_{R}-\omega_{0}^{\prime}\right)-\gamma_{r} / 2}{i\left(\omega_{R}-\omega_{0}^{\prime}\right)+\gamma_{r} / 2} S_{1, \text { out }},
$$

where $a_{c c}$ is the amplitude of the counterclockwise resonance, $\gamma_{r}$ is the energy decay constant of the magneto-optical resonator into the waveguide, and $\omega_{0}^{\prime}$ is the frequency of light. The phase reference of the field is at the center of the magneto-optical resonator. Similarly for the left-propagating light, which couples to the clockwise rotating resonance, we have

$$
S_{1, \text { in }}=\frac{i\left(\omega_{L}-\omega_{0}^{\prime}\right)-\gamma_{r} / 2}{i\left(\omega_{L}-\omega_{0}^{\prime}\right)+\gamma_{r} / 2} S_{2, \text { out }} .
$$

Since $\omega_{R}$ and $\omega_{L}$ are different due to the magneto-optical effect, the phases of the transmission for the two directions are asymmetric. We note since the resonators are apart enough such that there is no direct coupling between them through evanescent field, Eqs. (25) and (26) along with Eqs. (11)-(14) can be used to describe the three-resonator system of Fig. 3(b).

Suppose $\omega_{0}^{\prime}=\left(\omega_{L}+\omega_{R}\right) / 2$ and $\Delta \omega \leqslant \gamma_{r} / 2$, then using Eqs. (27) and (28) we have

$$
\phi_{L}-\phi_{R} \approx 4 \frac{\Delta \omega}{\gamma_{r}}, \quad \phi_{L}+\phi_{R}=2 \pi .
$$

Taking into account of the propagation phase $\phi_{s}$ in the short section of photonic crystal waveguide, which is reciprocal, and using Eqs. (19) and (20), we find the coupling between the two resonators to be

$$
V_{12}=-e^{i\left(\phi_{L}-\phi_{R}\right)} \frac{\gamma}{2 \sin \phi_{S}}
$$

And $V_{21}=V_{12}^{\star}$. By properly choosing the length of the waveguide section, we can tune $\phi_{s}=\pi / 2 \bmod (2 \pi)$.

\section{Two-dimensional lattice}

Now we can proceed to construct a resonator lattice which has a uniform effective magnetic field in a similar fashion as in Sec. III C. The structure is shown in Fig. 3(c), where the connection between two photonic crystal resonators along the $x$ axis is mediated by a magneto-optical resonator, while the connection between two resonators along the $y$ axis is through a short section of waveguide without a magnetooptical resonator. Magnetization is along the $z$ axis.

The coupling between two resonators along the $y$ axis is given by

$$
V_{r, r+a \hat{y}}=\frac{\gamma}{2} .
$$

Using Eq. (32), we find the coupling between two resonators along the $x$ axis to be

$$
V_{r, r+a \hat{x}}=-e^{i \phi(y)} \frac{\gamma}{2},
$$


where $\phi(y)=2 \Delta \omega(y) / \gamma_{r}$. Again, in order to implement the Landau gauge, the magnetization has a constant gradient along the $y$ axis. Thus, the frequency splitting is proportional to $y$, i.e., $\Delta \omega(y)=\Delta \omega_{m} y /\left(N_{y} a\right)$. As a result, the effective magnetic field is

$$
B_{\mathrm{eff}}=\frac{2 \Delta \omega_{m}}{\gamma_{r} N_{y} a^{2}}
$$

Suppose the magneto-optical resonator has an internal $Q$ factor of $10^{3}$ [defined as $\omega_{0} /\left(2 \gamma_{r}\right)$ ], then in order to achieve the maximum direction-dependent phase in the linear regime [29], which equals about 2 [when $\Delta \omega \approx \gamma_{r} / 2$ in Eq. (29)], the magneto-optical effect only needs to be such that $\epsilon_{i} / \epsilon_{r} \approx$ $1 /(4 Q)=2.5 \times 10^{-4}$, where we have used $\Delta \omega \sim \omega_{0} \epsilon_{i} / \epsilon_{r}$. Such a magnitude of the magneto-optical effect can be routinely achieved in bismuth iron garnet [30,31]. Comparing to the case without the magneto-optic resonator, the directiondependent phase difference is enhanced by about a factor of $Q$, with the same magneto-optic effect strength and length of waveguide. In a lattice with $N_{y}=100$, the maximum value for
$B_{\text {eff }}$ can approach $0.02 / a^{2}$, which is at least one order larger than the scheme without a magneto-optical resonator.

\section{CONCLUSION}

In summary, we make use of the magneto-optical effect to achieve effective magnetic field for photons in photonic crystal resonator lattices. The effective magnetic field arises from the direction-dependent phase for propagating photons. We propose two different realizations with the directiondependent coupling phase induced by the magneto-optical effect between two photonic crystal resonators, where the coupling is built upon the magneto-optical waveguide connection and the resonator connection respectively. We give estimations for the two realizations based on experimental data.

\section{ACKNOWLEDGMENTS}

This work was supported in part by US Air Force Office of Scientific Research Grant No. FA9550-09-1-0704, and US National Science Foundation Grant No. ECCS-1201914.
[1] M. Hafezi, E. A. Demler, M. D. Lukin, and J. M. Taylor, Nat. Phys. 7, 907 (2011).

[2] R. O. Umucalilar and I. Carusotto, Phys. Rev. A 84, 043804 (2011).

[3] K. Fang, Z. Yu, and S. Fan, Phys. Rev. Lett. 108, 153901 (2012).

[4] K. Fang, Z. Yu, and S. Fan, Nat. Photon. 6, 782 (2012).

[5] K. Fang, Z. Yu, and S. Fan, Phys. Rev. B 87, 060301(R) (2013).

[6] A. B. Khanikaev, S. H. Mousavi, W.-K. Tse, M. Kargarian, A. H. MacDonald, and G. Shvets, Nat. Mater. 12, 233 (2013).

[7] M. C. Rechtsman, J. M. Zeuner, A. Tünnermann, S. Nolte, M. Segev, and A. Szameit, Nat. Photon. 7, 153 (2013).

[8] M. C. Rechtsman, J. M. Zeuner, Y. Plotnik, Y. Lumer, D. Podolsky, F. Dreisow, S. Nolte, M. Segev, and A. Szameit, Nature (London) 496, 196 (2013).

[9] K. Fang, Z. Yu, and S. Fan, Opt. Express 21, 18216 (2013).

[10] K. Fang and S. Fan [Phys. Rev. Lett. (to be published)].

[11] F. D. M. Haldane and S. Raghu, Phys. Rev. Lett. 100, 013904 (2008).

[12] K. Fang, Z. Yu, and S. Fan, Phys. Rev. B 84, 075477 (2011).

[13] L. Lu, L. Fu, J. D. Joannouplos, and M. Soljacic, Nat. Photon. 7, 294 (2013).

[14] Z. Yu and S. Fan, Nat. Photon. 3, 91 (2009).

[15] H. Lira, Z. Yu, S. Fan, and M. Lipson, Phys. Rev. Lett. 109, 033901 (2012).

[16] J. D. Jackson, Classical Electrodynamics (Wiley, New York, 1998).

[17] Z. Wang, Y. Chong, J. D. Joannopoulos, and M. Soljacic, Nature (London) 461, 772 (2009).

[18] Z. Yu, G. Veronis, Z. Wang, and S. Fan, Phys. Rev. Lett. 100, 023902 (2008)

[19] J. M. Luttinger, Phys. Rev. 84, 814 (1951).

[20] R. L. Espinola, T. Izuhara, M.-C. Tsai, R. M. Osgood, Jr., and H. Dötsch, Opt. Lett. 29, 941 (2004).
[21] T. Shintaku, T. Uno, and M. Kobayashi, J. Appl. Phys. 74, 4877 (1993).

[22] W. Zhang, J.-W. Mu, W.-P. Huang, and W. Zhao, Appl. Phys. Lett. 98, 171109 (2011).

[23] A linearly varying magnetization can be achieved by placing the photonic crystal chip away from a wire with constant current, such that $L / R \ll 1$, where $L$ is the length of the chip and $R$ is the distance of the chip from the wire. If the chip is placed on the constant azimuthal angle plane, then we have linearly varying magnetic field perpendicular to the chip which can be used in the proposal of Sec. IV; if the chip is tilted a small angle with respect to the constant azimuthal angle plane, then we can obtain linearly varying magnetic field along the chip, which can be used here.

[24] Z. Wang and S. Fan, Phys. Rev. E 68, 066616 (2003).

[25] Z. Wang and S. Fan, Appl. Phys. B 81, 369 (2005).

[26] W. Smigaj, J. Romero-Vivas, B. Gralak, L. Magdenko, B. Dagens, and M. Vanwolleghem, Opt. Lett. 35, 568 (2010).

[27] In the presence of waveguide, the two degenerate modes of the magneto-optical resonator split even without magnetization. To recover the the degeneracy of the two modes in the absence of the magneto-optical effect, one cylinder near the waveguide is modified as shown in Fig. 3(b) [24].

[28] S. Fan, P. R. Villeneuve, J. D. Joannopoulos, and H. A. Haus, Phys. Rev. Lett. 80, 960 (1998).

[29] If one wants to use a larger magneto-optical effect beyond the linear phase-frequency regime, then a more complicated magnetization domain engineering in the lattice is needed to achieve uniform effective magnetic field while a constant gradient of magnetization does not suffice.

[30] N. Adachi, V. P. Denysenkov, S. I. Khartsev, and A. M. Grishin, J. Appl. Phys. 88, 2734 (2000).

[31] T. Tepper and C. A. Ross, J. Cryst. Growth 255, 324 (2003). 